\title{
Análisis del aprovechamiento de las exportaciones colombianas en el marco del TLC Colombia-Canadá
}

\author{
María Isabel Montoya-Durán ${ }^{1}$ \\ Universidad de La Salle \\ mariaimontoya88@unisalle.edu.co \\ Laura Tatiana Limas-Mendigaño ${ }^{2}$ \\ Universidad de La Salle \\ llimas12@unisalle.edu.co
}

DOI: https://doi.org/10.21158/23227230.v10.n0.2020.2877

Cómo citar este artículo: Montoya-Durán, M. I.; Limas-Mendigaño, L. T. (2020). Análisis del aprovechamiento de las exportaciones colombianas en el marco del TLC Colombia-Canadá. Revista Ploutos, 10, (Páginas). DOI: https://doi.org/10.21158/23227230.v10.n0.2020.2877

Fecha de recepción: 09 de septiembre de 2020

Fecha de aprobación: 25 de marzo de 2021

\section{Resumen}

El objetivo general de esta investigación es analizar el aprovechamiento de las exportaciones colombianas en el marco del TLC Colombia-Canadá, dado que este es el primer acuerdo celebrado entre Colombia y un país desarrollado de la región. El estudio utiliza una metodología mixta; inicialmente, a través de una revisión documental, se describe el comercio internacional de Colombia y Canadá, luego se identifican las tendencias de comercio bilateral entre los dos países en el 2018 y se caracterizan las condiciones arancelarias actuales con una matriz comparada de análisis. Finalmente, se analiza la oferta exportable colombiana frente a la demanda canadiense, con indicadores comerciales tales como el IVCR, el índice de intensidad comercial y el índice de complementariedad, a fin de identificar sectores en los que Colombia tenga ventaja frente a Canadá. El resultado de la investigación evidencia que Canadá no es un socio comercial significativo para Colombia, pero se puede aprovechar la ventaja comparativa en sectores como la floricultura, con el fin de aumentar las exportaciones al mercado canadiense.

Palabras clave: comercio internacional; tratado de libre comercio; indicadores comerciales; exportaciones colombianas; comercio bilateral.

1 Profesional en Finanzas y Comercio internacional de la Universidad La Salle. Integrante del semillero de SIAFCI. ORCID: https://orcid.org/0000-0002-0399-5803

2 Profesional en Finanzas y Comercio internacional de la Universidad La Salle. Integrante del semillero de SIAFCI. ORCID: ORCID: https://orcid.org/0000-0001-7191-3075 


\title{
Analysis of Colombian export harnessing within the framework of the Colombia-Canada Free Trade Agreement (FTA)
}

\begin{abstract}
The general objective of this research is to analyze the leverage of Colombian exports within the framework of the Colombia-Canada Free Trade Agreement (FTA), given that this is the first agreement being reached between Colombia and a developed country in the region. The study uses a mixed methodology; initially describing the international trade agreement between Colombia and Canada by means of a literature review; after that, we identified the bilateral trade trends between the two countries in 2018, and characterized the current tariff conditions with a comparative matrix analysis. Finally, we analyze the Colombian exportable supply against the Canadian demand, with trade indicators such as the IVCR, the trade intensity index, and the complementarity index, in order to identify the sectors in which Colombia has an advantage over Canada. The results of the research show that Canada is not a significant trading partner for Colombia, but it is possible to profit from the comparative advantage in sectors like floriculture, in order to increase exports to the Canadian market.
\end{abstract}

Keywords: international trade; free trade agreement; trade indicators; Colombian exports; bilateral trade.

\section{Análise do uso das exportações colombianas no marco do TLC Colômbia-Canadá}

\section{Resumo}

O objetivo geral desta pesquisa é analisar o uso das exportações colombianas no marco do TLC Colômbia-Canadá, visto que este é o primeiro acordo entre a Colômbia e um país desenvolvido da região. $O$ estudo usa uma metodologia mista, inicialmente, por meio de uma revisão documental, descreve-se o comércio internacional da Colômbia e do Canadá, em seguida são identificadas as tendências do comércio bilateral entre os dois países em 2018 e as atuais condições tarifárias são caracterizadas com uma matriz de análise comparativa. Finalmente, analisa-se a oferta exportável colombiana em relação à demanda canadense, com indicadores comerciais como o IVCR, o índice de intensidade comercial e o índice de complementaridade, para identificar os setores em que a Colômbia tenha vantagem sobre o Canadá. $O$ resultado da investigação mostra que o Canadá não é um sócio comercial significativo para a Colômbia, mas a vantagem comparativa pode ser aproveitada em setores como a floricultura, a fim de aumentar as exportações para o mercado canadense.

Palavras-chave: comércio internacional, tratado de Livre Comércio, indicadores de negócios, exportações colombianas, comércio bilateral. 
Analyse des bénéfices des exportations colombiennes dans le cadre de l'accord de libre échange (ALE) entre la Colombie et le Canada

\begin{abstract}
Résumé
L'objectif de cette étude est d'analyser les bénéfices obtenus pour les exportations colombiennes dans le cadre de l'ALE Colombie-Canada, dans la mesure où il s'agit du premier accord régional de ce type entre la Colombie et un pays développé. L'étude utilise une méthodologie mixte au travers d'une analyse documentaire du commerce colombo-canadien, puis identifie les grandes tendances de ce commerce bilatéral pour l'année 2018 et caractérise ensuite les conditions tarifaires actuelles à l'aide d'une matrice d'analyse comparative. Enfin, nous analyserons le type d'exportation colombienne en fonction de la demande canadienne grâce à des indicateurs commerciaux tels que l'IVCR, l'indice d'intensité commerciale et l'indice de complémentarité dans le but d'identifier les secteurs dans lesquels la Colombie a un avantage comparatif face au Canada. Le résultat de l'étude montre que le Canada n'est pas un partenaire commercial fondamental pour la Colombie, mais que l'avantage comparatif colombien devrait davantage être mis à profit dans des secteurs tels que la floriculture afin d'accroître les exportations vers le marché canadien.
\end{abstract}

Mots-clés: commerce international; traité de libre-échange; indicateurs commerciaux; exportations colombiennes; échange bilatéral.

\title{
1. Introducción
}

Desde el 2018, el Gobierno colombiano decidió no iniciar más negociaciones de tratados de libre comercio hasta lograr el aprovechamiento de cada uno de los acuerdos vigentes. Sin embargo, no hay estudios gubernamentales públicos que afirmen con certeza el grado de aprovechamiento de estos TLC.

A la fecha, son 15 los acuerdos o tratados celebrados por Colombia que posibilitan la expansión bajo mejores condiciones comerciales (Ministerio de Comercio, 2018). A partir del 15 de agosto del 2011 entró en vigor el TLC Colombia-Canadá (MinComercio, s. f.). Para ese año las exportaciones de Colombia hacia Canadá representaron el 1,08 \% del total de las ventas colombianas al exterior, y en el 2018 representaron el 2 \% (TradeMap, 2018), lo que cuestiona el aprovechamiento que se está realizando del TLC en términos de exportaciones. 
En el 2011 las exportaciones COL-CAN fueron de USD 614 -millones-, mientras que para el 2018 fueron de USD 797,568 - millones—, aumentando en siete años USD 183 —millones-. Si se tiene en cuenta que, a la fecha, aproximadamente el $98 \%$ del universo arancelario se encuentra desgravado y que Canadá es un país con alta capacidad de compra, debido a que su PIB per cápita es el segundo más alto de la región, Colombia podría aumentar sus exportaciones a partir del aprovechamiento de este TLC.

El TLC Colombia-Canadá, entonces, tiene potencial de ser mejor aprovechado por Colombia, en primer lugar, porque podría aumentar la diversificación de mercados, disminuyendo la concentración en países como Estados Unidos. En segundo lugar, mercados como Canadá ofrecen una oportunidad para Colombia, ya que brindan la posibilidad de un mayor aprovechamiento de exportaciones con productos con beneficios arancelarios, ventaja comparativa y oferta exportable. La investigación busca contribuir con el análisis de los flujos comerciales entre Colombia y Canadá antes y después de la firma del TLC, con el fin de establecer el aprovechamiento del acuerdo y proponer sectores para mejorar los niveles de exportaciones colombianas hacia Canadá.

\section{Metodología y datos}

Este estudio utilizará una metodología mixta que permita integrar herramientas de análisis cualitativo y cuantitativo. La investigación tiene un alcance descriptivo. La metodología mixta o la investigación multimétodo, según Pole (2009), utiliza la recolección y el análisis de datos cuantitativos y cualitativos en los métodos que forman parte del estudio.

La investigación se aborda con un diseño no experimental. El diseño de investigación no experimental se define como la investigación que se realiza sin una manipulación deliberada de variables (Hernández-Sampieri, 2017). Las variables utilizadas en la investigación son las exportaciones bilaterales totales Colombia-Canadá por capítulo de la partida arancelaria en el 2018 y las condiciones arancelarias vigentes por subpartida 
arancelaria en el 2018; estos datos se obtienen de Trademap y del acuerdo oficial del TLC Colombia-Canadá.

Las exportaciones se definen como la salida del territorio aduanero nacional de mercancías para su uso o consumo definitivo en otro país (DANE, 2017). Las condiciones arancelarias son las establecidas en el TLC para la eliminación progresiva y cronológica de aranceles aduaneros establecidos en el marco de la firma de un TLC; los aranceles son los derechos de aduanas que se aplican a las importaciones de mercancías (Procolombia, 2016). Las mercancías producto de exportación tienen una codificación numérica que se basa en el Sistema Armonizado. Esta codificación se denomina partida arancelaria y está dividida en capítulos — dos primeros dígitos de la codificación numérica—, partidas cuatro primeros dígitos de la codificación numérica- y subpartidas arancelarias — seis primeros dígitos de la codificación numérica-.

Inicialmente, se presenta la descripción del comercio exterior de Colombia y Canadá para identificar los principales socios comerciales y la situación general de su comercio de bienes. Posteriormente, se caracteriza el comercio bilateral entre ambos países y los productos objeto de comercio internacional en el 2018, para lo cual se utiliza la técnica de revisión documental a partir de cifras obtenidas de entidades y bases de datos del Ministerio de Comercio, Industria y Turismo, ProColombia, TradeMap y LegisComex, entre las principales. A continuación, se presenta estadística descriptiva utilizada para identificar tendencias en el comportamiento del comercio bilateral; con este fin se utilizaron tasas de participación y tasa de crecimiento compuesto con el método CAGR - compound annual growth rate-.

La caracterización de las canastas de desgravación se realiza a partir de la elaboración de una matriz comparada de categorías de análisis con información en la que se analiza el número de partidas arancelarias contenida en cada canasta, el periodo de desgravación, el sector económico al que pertenece y el valor porcentual desgravado, entre 
los principales. Los resultados se presentan gráficamente identificando la concentración de sectores dentro de cada una de las canastas de desgravación y el valor del gravamen de acuerdo con el tiempo negociado de desgravación en cada canasta.

Por último, para el desarrollo del tercer objetivo, esto es, identificar los sectores con mayor potencialidad para Colombia frente al comercio con Canadá, y con el fin de determinar oportunidades que permitan mejorar el aprovechamiento del TLC, se parte de indicadores comerciales como herramienta de análisis cualitativo. Dentro de los índices descritos por el Banco Mundial (2010) que se utilizaron a lo largo de la investigación se encuentran los que se enlistan y describen a continuación.

- Índice de ventaja comparativa revelada (IVCR). Evalúa el potencial de exportación de un país $i$ para un producto $j$ aportando información útil acerca de las posibles perspectivas comerciales con un nuevo asociado; se calcula de la siguiente forma:

$$
I C V R i j=\frac{x i j}{X i t} /_{\frac{x w j}{X w t}}(1)
$$

- Índice de intensidad comercial (T). Determina si el valor del comercio entre dos países es mayor o menor de lo que podría esperarse sobre la base de su importancia en el comercio mundial. La fórmula del índice es:

$$
T i j=\frac{x i j}{X i t} / \frac{x w j}{X w t}(2)
$$

- Índice de complementariedad (CC). Evidencia qué tan bien coinciden las estructuras de importación y exportación de un país. El CC entre los países $k$ y $j$ se define como:

$$
C C i j=100 *\left(1-\sum \frac{[m i k-x i j]}{2}\right)
$$


Después de la identificación del sector con mayor potencial para las exportaciones colombianas se realiza una proyección mensual con miras al 2020, es decir, por un periodo de 12 meses. La recolección de datos se realiza de fuente secundaria a través de bases de datos de Trademap —organización que obtiene los datos de la Dian y de Stadistics Canadáy datos obtenidos del acuerdo oficial del TLC Colombia-Canadá.

El método de recolección de datos utilizado para la investigación es el uso de bases de datos. En la primera parte se utiliza la información de las importaciones y exportaciones totales entre Colombia-Mundo y Canadá-Mundo, posteriormente, la base de datos de comercio bilateral Colombia-Canadá en el 2018 por subpartida arancelaria y se le adiciona una columna con los datos de las condiciones arancelarias, a fin de evidenciar el comercio actual y qué productos son objeto de intercambio entre estos países. Con esta información se aplican diferentes índices comerciales —intensidad comercial, complementariedad y ventaja comparativa en las exportaciones a Canadá - con el fin de identificar los sectores en los que Colombia tiene ventaja para las exportaciones.

En la segunda parte, luego de identificar el sector potencial de exportaciones colombianas, se realiza una base de datos con las exportaciones de dicha partida en una serie de tiempo mensual desde el 2005, que se compone de 179 observaciones, con el propósito de realizar una proyección de las exportaciones para el 2020.

\section{Resultados}

Los resultados de la investigación están dados en el orden del desarrollo y del abordaje de estos, por lo cual se presentan de acuerdo con los objetivos específicos derivados del objetivo general que corresponde al análisis del aprovechamiento de las exportaciones colombianas en el marco del TLC Colombia-Canadá. 


\subsection{Objetivo 1: caracterizar el comercio exterior de Colombia y Canadá}

Para la descripción del comercio exterior de Colombia y Canadá, teniendo en cuenta la regla de 80/20 o de Pareto, se crea un top 10 para el análisis de socios comerciales, importaciones, exportaciones, participación en las exportaciones mundiales, concentración de exportación y balanzas comerciales superavitarias de cada país y para el comercio bilateral entre Colombia-Canadá. Esta caracterización se realiza usando herramientas de Excel y R Studio con base en datos de Trademap y del TLC oficial Colombia-Canadá.

\subsubsection{Comercio exterior Colombia.}

Las exportaciones colombianas están altamente concentradas en el mayor socio comercial para el país, que es Estados Unidos. Es posible ver que Colombia tiene una tendencia de exportaciones a los países que son geográficamente más cercanos, lo que se evidencia al encontrar que el top 10 lo ocupan países como Estados Unidos, Panamá, Ecuador, México, Chile y Perú. A pesar de la cercanía geográfica, Canadá se encuentra en el puesto 12. En lo que tiene que ver con exportaciones, Colombia ha sido un país que se ha centrado en la exportación de hidrocarburos que corresponden al sector de minerales, químicos y plásticos, con tan solo una participación del $1 \%$ en el escenario mundial.

Tabla 1. Destinos de las exportaciones colombianas 2018

\begin{tabular}{|c|c|c|c|} 
Posición & Importadores & $\begin{array}{c}\text { ValorUSD } \\
\text { exportadoen 2018 }\end{array}$ & Participación \\
\hline 1 & Estados Unidos de América & 11.080 & $27 \%$ \\
\hline 2 & China & 3.748 & $9 \%$ \\
\hline 3 & Panamá & 3.597 & $9 \%$ \\
\hline 4 & Ecuador & 1.851 & $4 \%$ \\
\hline 5 & Turquía & 1.691 & $4 \%$ \\
\hline 6 & México & 1.638 & $4 \%$ \\
\hline 7 & Brasil & 1.561 & $4 \%$ \\
\hline 8 & Chile & 1.190 & $3 \%$ \\
\hline 9 & España & 1.190 & $3 \%$ \\
\hline 10 & Perú & 1.165 & $3 \%$ \\
\hline 11 & Países Bajos & 1.034 & $2 \%$ \\
\hline 12 & Canadá & 797 & $2 \%$ \\
\hline & Mundo & 41.769 & $100 \%$ \\
\hline
\end{tabular}

Fuente. Elaboración propia con información de TradeMap. 
La diversificación de la oferta exportable colombiana es baja. Es posible ver que, en segundo lugar, pero con un saldo muy por debajo del sector de hidrocarburos - USD 24163 millones - se encuentra el café - USD 2348 millones - que hace parte sector agroindustrial colombiano con un 4,9 \% en la participación mundial.

Tabla 2. Productos exportados por Colombia, 2018

\begin{tabular}{|c|c|c|c|c|} 
Orden & Código & Descripción del producto & $\begin{array}{c}\text { Valor exportado } \\
\text { en 2018 }\end{array}$ & Participación \\
\hline 1 & 27 & $\begin{array}{c}\text { Combustibles minerales, aceites minerales y productos de su } \\
\text { destilación. }\end{array}$ & 24.163 & $58 \%$ \\
\hline 2 & 9 & Café, té, yerba mate y especias. & 2.348 & $6 \%$ \\
\hline 3 & 71 & $\begin{array}{c}\text { Perlas finas, piedras preciosas o semipreciosas, metales } \\
\text { preciosos. }\end{array}$ & 1.632 & $4 \%$ \\
\hline 4 & 39 & Plástico y sus manufacturas & 1.532 & $4 \%$ \\
\hline 5 & 6 & Plantas vivas y productos de la floricultura & 1.477 & $4 \%$ \\
\hline 6 & 8 & $\begin{array}{c}\text { Frutas y frutos comestibles; cortezas de agrios (cítricos), melones } \\
\text { o sandías }\end{array}$ & 1.057 & $3 \%$ \\
\hline 7 & 72 & Fundición, hierro y acero & 650 & $2 \%$ \\
\hline 8 & 87 & $\begin{array}{c}\text { Vehículos automóviles, tractores, velocípedos y demás vehículos } \\
\text { terrestres, sus partes y accesorios }\end{array}$ & 645 & $2 \%$ \\
\hline 9 & 15 & Grasas y aceites animales o vegetales, grasas alimenticias. & 623 & $1 \%$ \\
\hline 10 & 17 & Azúcares y artículos de confitería & 518 & $1 \%$ \\
\hline \multicolumn{2}{|c|}{ TOTAL } & Todos los productos & 41.769 & $100 \%$ \\
\hline
\end{tabular}

Fuente. Elaboración propia con información de TradeMap.

Las mayores participaciones de productos exportados por Colombia hacia el mundo están concentradas en el sector agroindustrial, en el cual se destaca la floricultura y el café con el mayor rango en el ámbito mundial. Tan solo tres productos están por encima de los hidrocarburos, lo que vuelve a cuestionar la diferenciación en la oferta exportable colombiana.

Para las importaciones colombianas el principal socio comercial es Estados Unidos, país con el cual entró en vigencia un tratado de libre comercio a partir del 15 de mayo del 2012 con la publicación del Decreto 993. México cuenta con un tratado de libre comercio con Colombia desde el 1 de enero de 1995, el 2 de agosto del 2011 entra en vigencia una 
profundización y adecuación del TLC. Canadá se encuentra en el puesto 11 en importaciones hacia Colombia para el 2018 a pesar del TLC vigente.

Tabla 3. Principales países proveedores de las importaciones colombianas, 2018

\begin{tabular}{|c|c|c|c|}
\hline Posición & Exportadores & $\begin{array}{c}\text { ValorUSD } \\
\text { importado en 2018 }\end{array}$ & Participación \\
\hline 1 & Estados Unidos de América & 13.075 & $26 \%$ \\
\hline 2 & China & 10.544 & $21 \%$ \\
\hline 3 & México & 3.947 & $8 \%$ \\
\hline 4 & Brasil & 2.828 & $6 \%$ \\
\hline 5 & Alemania & 2.173 & $4 \%$ \\
\hline 6 & Japón & 1.288 & $3 \%$ \\
\hline 7 & India & 1.183 & $2 \%$ \\
\hline 8 & Francia & 1.172 & $2 \%$ \\
\hline 9 & España & 991 & $2 \%$ \\
\hline 10 & Zona franca & 938 & $2 \%$ \\
\hline 11 & Canadá & 845 & $2 \%$ \\
\hline & Mundo & 51.232 & $100 \%$ \\
\hline
\end{tabular}

Fuente. Elaboración propia con información de TradeMap.

\subsubsection{Comercio exterior Canadá.}

El principal socio comercial de Canadá es Estados unidos, con el cual se encuentra geográficamente cerca, lo que facilita la logística de intercambios comerciales. Con la información proporcionada en la tabla se evidencia que del continente americano tan solo se encuentra Estados Unidos y México. Para el 2018 Colombia exportó a Canadá tan solo USD 797 millones. Esto a pesar de que con los dos países Colombia goza de un TLC.

Las exportaciones canadienses también tienen como destino principal Estados Unidos. En América solo se encuentra como otro mercado importante receptor de la mercancía producida en Canadá a México. 
Los principales productos importados por Canadá corresponden al sector industrial y al sector de minerales, químicos y plásticos. En primer lugar, vehículos automóviles, tractores, velocípedos y demás vehículos terrestres, sus partes y accesorios.

Tabla 4. Principales países proveedores de las importaciones canadienses, 2018

\begin{tabular}{|c|c|c|c|}
\hline Posición & Exportadores & $\begin{array}{r}\text { Valorimportado USD } \\
\text { en 2018 }\end{array}$ & Participación \\
\hline 1 & Estados Unidos de América & 235.151 & $51 \%$ \\
\hline 2 & China & 58.263 & $13 \%$ \\
\hline 3 & México & 28.398 & $6 \%$ \\
\hline 4 & Alemania & 14.708 & $3 \%$ \\
\hline 5 & Japón & 13.005 & $3 \%$ \\
\hline 6 & Corea, República de & 7.282 & $2 \%$ \\
\hline 7 & Italia & 6.942 & $2 \%$ \\
\hline 8 & Reino Unido & 7.100 & $2 \%$ \\
\hline 9 & Francia & 5.545 & $1 \%$ \\
\hline 10 & Viet Nam & 4.152 & $1 \%$ \\
\hline & Colombia & 797 & $0.2 \%$ \\
\hline & Mundo & 459.839 & $100 \%$ \\
\hline
\end{tabular}

Fuente. Elaboración propia con información de TradeMap.

Canadá cuenta con una balanza comercial con saldo superavitario para la mayor parte de socios comerciales importantes del país, por ejemplo, su mayor socio Estados Unidos tiene un saldo positivo. La mayor participación de Canadá en las exportaciones mundiales se encuentra en los productos derivados del sector de manufacturas, que para el 2018 rondaba el 12 \% e, incluso, alcanzó el $14 \%$ en algunos productos derivados de este sector. Otros sectores como el agro, puntualmente con productos como abonos, semillas y frutos, cereales, hortalizas, plantas y tubérculos alimenticios, tienen una alta capacidad de producción, por lo que su participación en el comercio mundial está por encima del 5 \%. 


\subsubsection{Comercio bilateral Colombia-Canadá.}

Para el análisis del comercio bilateral entre Colombia-Canadá se propone que, al tener como referencia el 2011, se analice la situación inicial del comercio cuando se puso en vigencia el acuerdo y la situación actual, año 2018, a fin de analizar los cambios en el flujo bilateral de mercancías.

Al analizar los productos que se están comercializando se observa que no han tenido cambios radicales, ya que en importaciones el trigo, y puntualmente el sector agrícola, tiene aún el primer lugar, así como el sector industrial es aún fuerte en lo que respecta a las importaciones de Colombia desde Canadá. El café, también perteneciente al sector agrícola, para el 2018 se encuentra en segundo lugar en exportaciones con la única variación del oro, ubicado en primer lugar en el 2018, el cual en el 2011 no se encontraba en los primeros lugares de exportación.

Así, por ejemplo, en el 2011 se encontraba en primer lugar el trigo blanco y los demás trigos con una participación de USD 185 millones y pasó a USD 310 millones en el 2018. En el 2018 la cebada para elaboración de cerveza, que estuvo en el puesto 8 de las importaciones de Colombia desde Canadá en el 2011, ya no aparece en el top 10.

El sector de los minerales, químicos y plástico, además de las flores, que pertenecen al sector agroindustrial, tienen aún gran importancia en las exportaciones colombianas a Canadá. Para un mejor análisis de la situación del comercio entre Colombia y Canadá es posible analizar las variaciones del comercio en los dos periodos de análisis: el primero antes de la entrada en vigencia del TLC $\Delta \%$ 2005-2011 y el segundo desde que entro la vigencia del TLC $\Delta \%$ 2012-2018. Los resultados se resumen en la figura 1. 
Figura 1. Comercio bilateral Colombia-Canadá, 2018

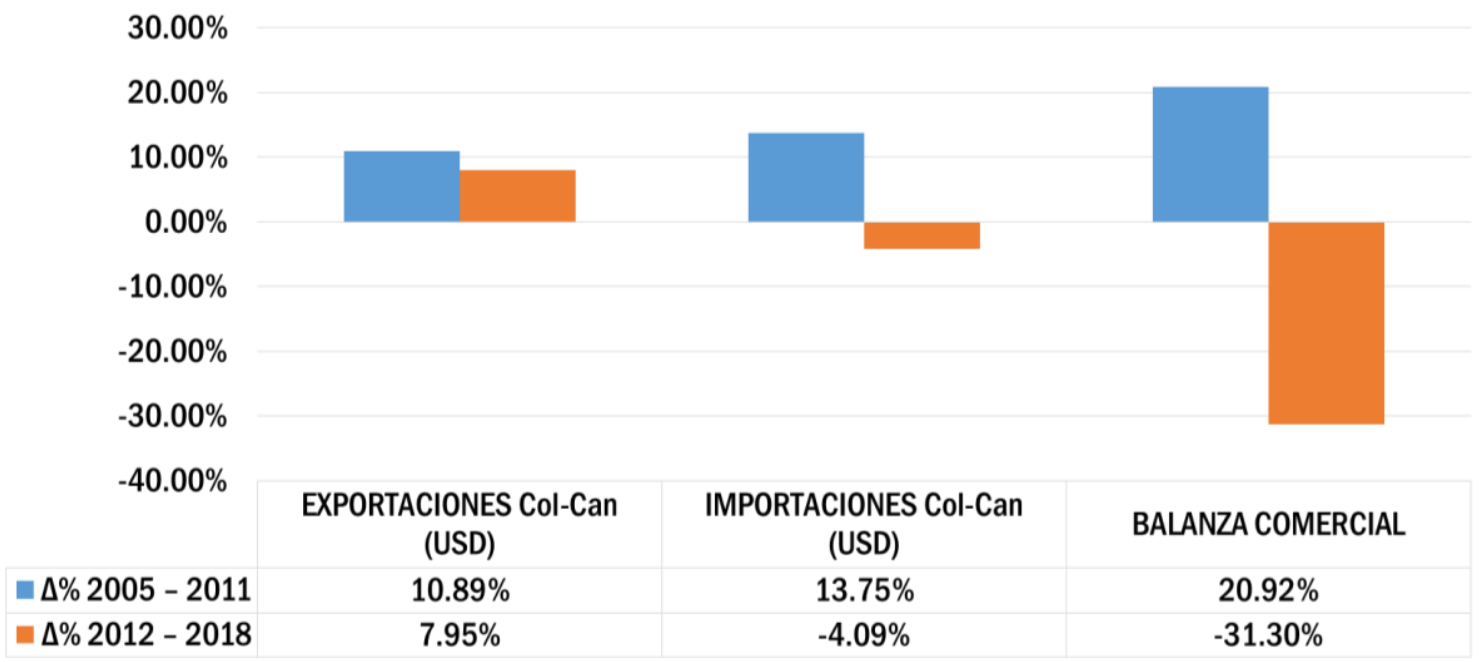

Fuente. Elaboración propia.

\subsubsection{Exportaciones colombianas dirigidas a Canadá.}

Tabla 5. Principales países destinos de exportaciones canadienses, 2018

\begin{tabular}{|c|c|c|c|c|}
\hline Orden & Código & Descripción delproducto & $\begin{array}{c}\text { Valor exportado } \\
\text { en } 2018\end{array}$ & Participación \\
\hline 1 & 27 & $\begin{array}{c}\text { Combustibles minerales, aceites minerales y productos de su } \\
\text { destilación. }\end{array}$ & 266 & $33 \%$ \\
\hline 2 & 71 & $\begin{array}{c}\text { Perlas finas, piedras preciosas o semipreciosas, metales } \\
\text { preciosos. }\end{array}$ & 222 & $28 \%$ \\
\hline 3 & 9 & Café, té, yerba mate y especias. & 181 & $23 \%$ \\
\hline 4 & 6 & Plantas vivas y productos de la floricultura & 41 & $5 \%$ \\
\hline 5 & 73 & Manufacturas de fundición, de hierro o acero & 26 & $3 \%$ \\
\hline 6 & 39 & Plástico y sus manufacturas & 9 & $1 \%$ \\
\hline 7 & 17 & Azúcares y artículos de confitería & 6 & $1 \%$ \\
\hline 8 & 8 & $\begin{array}{l}\text { Frutas y frutos comestibles; cortezas de agrios (cítricos), } \\
\text { melones o sandías }\end{array}$ & 5 & $1 \%$ \\
\hline 9 & 62 & $\begin{array}{c}\text { Prendas y complementos (accesorios), de vestir, excepto los } \\
\text { de punto }\end{array}$ & 3 & $0 \%$ \\
\hline 10 & 85 & $\begin{array}{c}\text { Máquinas, aparatos y material eléctrico, aparatos de } \\
\text { grabación o reproducción. }\end{array}$ & 3 & $0 \%$ \\
\hline \multicolumn{2}{|c|}{ TOTAL } & Todos los productos & 797 & $100 \%$ \\
\hline
\end{tabular}

Fuente. Elaboración propia con información de TradeMap. 
Al contrastar la información se puede caracterizar el comercio bilateral ColombiaCanadá con el fin de observar qué dinámicas han cambiado desde la entrada en vigencia del acuerdo comercial. Los resultados muestran que, a pesar de que desde el 2011 el tratado de libre comercio comenzó su implementación, el comercio bilateral no muestra mayores cambios, ya que en Colombia, por ejemplo, la oferta exportable es todavía liderada por los hidrocarburos.

Para el 2011 el 87 \% de las exportaciones colombianas se concentraban en tres tipos de productos: petróleo - capítulo 27-, café-capítulo 09-y plantas vivas-capítulo 06; en el 2018, ya con el TLC en vigencia y con el $98 \%$ de los productos desgravados, el $89 \%$ de las exportaciones las componen petróleo-capítulo 27—, perlas finas — capítulo 07-, café - capítulo 09- y plantas vivas - capítulo 06-, lo cual muestra que las exportaciones colombianas mantienen un nivel de concentración muy alto en sus ventas hacia Canadá. Además, son productos con bajo nivel de transformación: la media de exportaciones para este año fue de USD 8 millones.

En un periodo de aproximadamente nueve años el flujo de comercio bilateral no ha incrementado de manera significativa, ni estos dos países han reforzado el flujo comercial para ser socios actualmente fuertes.

3.1.5 Índice de intensidad comercial (T).

Tabla 6. Índice intensidad comercial Colombia-Canadá

\begin{tabular}{|c|c|c|c|c|}
\hline X COL-CAN & X MUNDO-CAN & $\begin{array}{r}\text { X TOTALES } \\
\text { COLOMBIA }\end{array}$ & X TOTALES MUNDO & INDICE \\
\hline USD 797 & USD 459.839 & USD 41.769 & USD 19.284.580 & 0,80 \\
\hline
\end{tabular}

Fuente. Elaboración propia, índice calculado con información de TradeMap. 
El índice determina si el valor del comercio entre dos países es mayor o menor de lo que podría esperarse sobre la base de su importancia en el comercio mundial. En este caso podemos decir que el flujo comercial es menor a lo esperado, ya que está por debajo de 1.

3.1.6 Índice de complementariedad (CC).

Proporciona información sobre las perspectivas de comercio intrarregional. Evidencia qué tan bien coinciden las estructuras de importación y exportación de un país. El índice es 0 cuando no se exportan bienes de parte de un país ni se importan por parte del otro, y 100 cuando las proporciones de exportaciones e importaciones coinciden con exactitud (Banco Mundial, 2010). En los resultados podemos ver las coincidencias en exportaciones por partida arancelaria del comercio Colombia-Canadá.

Tabla 7. Índice complementariedad comercial Colombia-Canadá

\begin{tabular}{|c|c|c|c|}
\hline Orden & Código del producto & Descripción del producto & Indice \\
\hline 1 & '27 & $\begin{array}{c}\text { Combustibles minerales, aceites minerales y productos de su destilación; } \\
\text { materias bituminosas; ... }\end{array}$ & $24,92 \%$ \\
\hline 2 & '87 & $\begin{array}{l}\text { Vehículos automóviles, tractores, velocípedos y demás vehículos terrestres, } \\
\text { sus partes y accesorios }\end{array}$ & $7,43 \%$ \\
\hline 3 & '84 & $\begin{array}{c}\text { Máquinas, aparatos y artefactos mecánicos, reactores nucleares, calderas; } \\
\text { partes de estas máquinas ... }\end{array}$ & $6,97 \%$ \\
\hline 4 & '85 & $\begin{array}{c}\text { Máquinas, aparatos y material eléctrico, y sus partes; aparatos de grabación } \\
\text { o reproducción ... }\end{array}$ & $4,33 \%$ \\
\hline 5 & '09 & Café, té, yerba mate y especias & $2,63 \%$ \\
\hline 6 & '06 & Plantas vivas y productos de la floricultura & $1,72 \%$ \\
\hline 7 & '90 & $\begin{array}{l}\text { Instrumentos y aparatos de óptica, fotografía o cinematografía, de medida, } \\
\text { control o precisión; ... }\end{array}$ & $1,26 \%$ \\
\hline 8 & '99 & Materias no a otra parte especificadas & $1,04 \%$ \\
\hline 9 & $' 71$ & $\begin{array}{c}\text { Perlas finas (naturales) o cultivadas, piedras preciosas o semipreciosas, } \\
\text { metales preciosos, ... }\end{array}$ & $0,99 \%$ \\
\hline 10 & '30 & Productos farmacéuticos & $0,94 \%$ \\
\hline
\end{tabular}

Fuente. Elaboración propia; índice calculado con información de TradeMap. 


\subsection{Objetivo 2: caracterizar las canastas de desgravación negociadas en el TLC}

El tratado de libre comercio celebrado entre Colombia y Canadá considera una lista de desgravación propuesta por Canadá y dos listas de desgravación propuestas por Colombia: una de productos agrícolas y otra de productos no agrícolas. Esto debido al especial tratamiento que se le da al sector agrícola en el país. Dado el objeto de estudio de esta investigación, únicamente se tendrá en cuenta la lista de desgravación de los productos canadienses.

A partir de la lista mencionada, contenida en el texto del acuerdo comercial, se logró identificar la concentración de sectores por canastas de desgravación. Para esto fue necesario identificar los capítulos de cada ítem del universo arancelario logrando así definir los sectores económicos a los que pertenece cada capítulo. Posteriormente, y para mejor interpretación y facilidad de análisis de gráficos, se reorganizaron en grupos económicos determinados a partir de una generalización del sector económico identificado en cada capítulo. Dado lo anterior, se obtuvo los resultados que se presentan en la tabla 8.

Tabla 8. Organización de partidas arancelarias

\begin{tabular}{|c|c|c|} 
CAPÍTULOS & SECTOR ECONÓMICO & GRUPO ECONÓMICO \\
\hline $1-10$ & Agrícola - Agropecuario & Agroindustria \\
$11-24$ & Agroindustria (productos procesados) & Minerales \\
\hline $25-27$ & Industrias químicas & Minerales, químicos y \\
$28-38$ & plástico & \\
$39-40$ & Cuero y Manufacturas \\
\hline $41-43,64$ & Manufacturas diversas & Manufacturas \\
$44-49,65-70,90-92,95-96$ & Textil y Confección & \\
$50-63$ & Joyería y Bisutería & \\
71 & Metalmecánica & \\
\hline $72-83$ & Máquinas y aparatos & Industrial \\
84,85 & Automotriz & \\
$86-89$ & Otros & \\
$93,97,98$ & Muebles & \\
94 & &
\end{tabular}

Fuente. Elaboración propia con información del TLC Colombia-Canadá. 
La lista de desgravación de Canadá contiene cinco canastas de desgravación cuyas composiciones y tiempos de desgravación se describen en la tabla 9.

Tabla 9. Canastas de desgravación arancelaria

\begin{tabular}{|c|c|c|}
$\begin{array}{c}\text { CANASTA DE } \\
\text { DESGRAVACIÓN }\end{array}$ & $\begin{array}{c}\text { CANTIDAD DE PARTIDAS } \\
\text { ARANCELARIAS }\end{array}$ & MECANISMO DE DESGRAVACIÓN \\
\hline A & 8138 & $\begin{array}{c}\text { Desgravación inmediata a partir de la fecha de entrada en vigor del } \\
\text { acuerdo comercial (año 2011) }\end{array}$ \\
\hline B & 19 & $\begin{array}{c}\text { Desgravación en tres etapas anuales iguales desde la entrada en vigor } \\
\text { del acuerdo comercial (desgravado totalmente en el año 2013) }\end{array}$ \\
\hline C & 156 & $\begin{array}{c}\text { Desgravación en 7 etapas anuales iguales desde la entrada en vigor del } \\
\text { acuerdo comercial (desgravado totalmente en el año 2017) }\end{array}$ \\
\hline D17 & 1 & $\begin{array}{c}\text { Desgravación en 17 etapas anuales iguales desde la entrada en vigor } \\
\text { del acuerdo comercial (desgravado totalmente en el año 2027) }\end{array}$ \\
\hline E & 110 & Exceptuadas de eliminación arancelaria \\
\hline TOTAL & 8424 &
\end{tabular}

Fuente. Elaboración propia con información del TLC Colombia-Canadá.

Como resultado de la caracterización de la lista de desgravación de Canadá se obtuvo la gráfica de la figura 2. 
Figura 2. Lista de desgravación Canadá

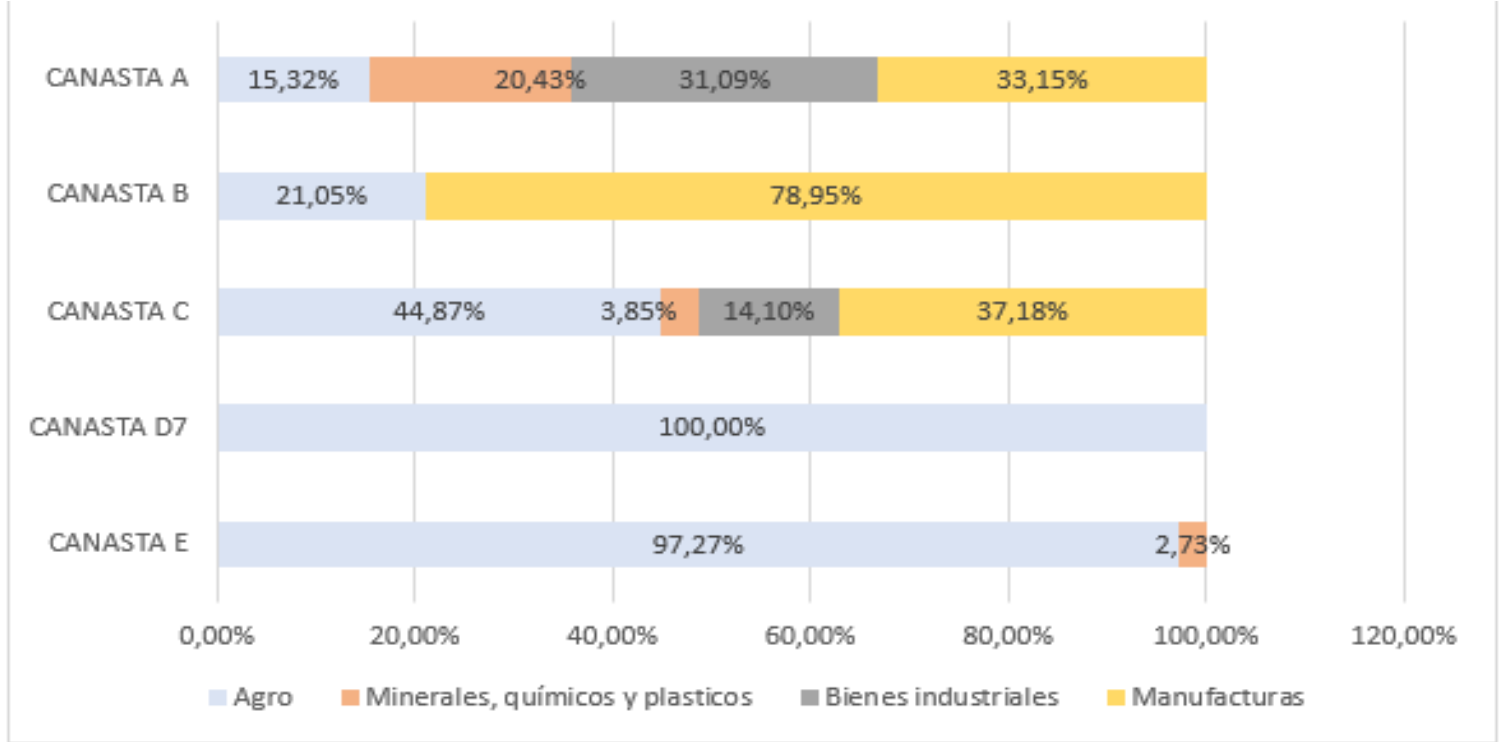

Fuente. Elaboración propia con información del acuerdo comercial Colombia-Canadá.

Dentro de la canasta A se encuentra contenido el 96,06 \% de los productos -8424 productos considerados en la lista de desgravación de Canadá-; esto es un indicio de la apertura del mercado canadiense al permitir la desgravación inmediata de la mayoría de los bienes. Aunque la canasta B es una canasta de desgravación a tres años, a la fecha se encuentra totalmente desgravada.

Su mecanismo de desgravación consistió en una desgravación paulatina dividida en tres etapas iguales anuales a partir de la fecha de entrada en vigor del acuerdo, con el fin de que para el año tres ya estuvieran todos los bienes totalmente desgravados. Dentro de esta canasta tan solo se contempló el 0,59 \% del grupo agroindustrial restante y el 6,05 \% de las manufacturas.

Para el caso de la canasta C, el mecanismo de desgravación también consistió en una eliminación arancelaria paulatina, pero dividida en siete etapas anuales iguales, con el fin de que para el año siete todos los bienes comprendidos en esta categoría estuvieran desgravados, es decir, a la fecha los bienes comprendidos en la canasta de desgravación C 
ya se encuentran totalmente desgravados. Aunque su composición es, principalmente, del grupo agroindustria, esta categoría, dentro de la canasta de desgravación C, tan solo representa el 8,01 \% del total de dicho grupo.

La canasta de desgravación a 17 años contiene los productos más protegidos por la industria canadiense, estos son los contenidos en la partida arancelaria 17019900, la cual corresponde a «azúcar de caña o remolacha y sacarosa químicamente pura, sólidos —con excepción de los que tienen adición de aromatizante o colorante, así como el azúcar en bruto-». Su mecanismo de desgravación es paulatino en 17 etapas anuales iguales.

La canasta E contiene los productos que están exentos de eliminación arancelaria y, aunque en su mayoría sean del grupo agro, representan el 15,22 \% del total de las partidas arancelarias consideradas en la lista de desgravación de Canadá, mientras que el 2,73 \% de los minerales, químicos y plásticos de esta canasta representan tan solo el 0,27 \% del total de dicho grupo.

Dentro de estos, se encuentran algunos productos comprendidos en los capítulos: 1. Animales vivos, 2. Carne y despojos comestibles, 4. Leche y productos lácteos, huevos de ave, miel natural, productos comestibles de origen animal no expresados ni comprendidos en otra parte, 15. Grasas y aceites animales o vegetales, productos de su desdoblamiento, grasas alimenticias elaboradas, ceras de origen animal o vegetal, 16. Preparaciones de carne, pescado o de crustáceos, moluscos o demás invertebrados acuáticos, 17. Azúcares y artículos de confitería, 18. Cacao y sus preparaciones, 19. Preparaciones a base de cereales, harina, almidón, fécula o leche, productos de pastelería, 21. Preparaciones alimenticias diversas, 22. Bebidas, líquidos alcohólicos y vinagres, 23. Residuos y desperdicios de las industrias alimentarias, alimentos preparados para animales y 35. Materias albuminoideas, productos a base de almidón o de fécula modificados, colas, enzimas. 


\subsection{1 Índice de ventaja comparativa revelada (IVCR)}

Evalúa el potencial de exportación de un país; es la comparación de dos partidas arancelarias entre dos países o un país o el resto del mundo.

Tabla 10. Índice de venta comparativa revelada

\begin{tabular}{|c|c|c|c|}
\hline Orden & Código del producto & Descripción del producto & Î́ndice \\
\hline 1 & '06 & Plantas vivas y productos de la floricultura & 29,74 \\
\hline 2 & '09 & Café, té, yerba mate y especias & 21,21 \\
\hline 3 & 17 & Azúcares y artículos de confitería & 4,69 \\
\hline 4 & '08 & Frutas y frutos comestibles; cortezas de agrios (cítricos), melones o sandías & 3,66 \\
\hline 5 & '27 & $\begin{array}{c}\text { Combustibles minerales, aceites minerales y productos de su destilación; } \\
\text { materias bituminosas; ... }\end{array}$ & 2,75 \\
\hline 6 & '15 & $\begin{array}{c}\text { Grasas y aceites animales o vegetales; productos de su desdoblamiento; } \\
\text { grasas alimenticias ... }\end{array}$ & 1,54 \\
\hline 7 & '41 & Pieles (excepto la peletería) y cueros & 1,02 \\
\hline 8 & '21 & Preparaciones alimenticias diversas & 0,85 \\
\hline 9 & '33 & $\begin{array}{l}\text { Aceites esenciales y resinoides; preparaciones de perfumería, de tocador o } \\
\text { de cosmética }\end{array}$ & 0,83 \\
\hline 10 & 170 & Vidrio y sus manufacturas & 0,72 \\
\hline 11 & '34 & $\begin{array}{c}\text { Jabones, agentes de superficie orgánicos, preparaciones para lavar, } \\
\text { preparaciones lubricantes, ... }\end{array}$ & 0,69 \\
\hline 12 & '69 & Productos cerámicos & 0,64 \\
\hline 32 & '45 & Corcho y sus manufacturas & \\
\hline
\end{tabular}

Fuente. Elaboración propia, índice calculado con información de TradeMap.

Es posible observar que el capítulo arancelario con mayor potencial de exportaciones para Colombia es el 06, que corresponde a plantas vivas y productos de la floricultura.

\subsection{Objetivo 3: identificar los sectores con mayor potencialidad para Colombia}

La identificación de los sectores con mayor potencialidad para Colombia se hace a partir del desarrollo del objetivo 1 y 2, en el cual se realizó un análisis del comercio bilateral Colombia-Canadá con bases de datos y usando una serie de índices comerciales. Se obtiene puntualmente con el índice de ventaja comparativa y revela que el sector agrícola y agroindustrial cuenta con la mayor ventaja para las exportaciones colombianas en el marco del TLC.

Esta identificación de los sectores y de las partidas arancelarias con mayor ventaja posibilita el aprovechamiento para el aumento de exportaciones colombianas al mercado 
canadiense; como resultado se tiene que el sector o capítulo arancelario de mayor potencial sería el 06, el cual es el correspondiente a plantas vivas y productos de la floricultura.

Con base en que las plantas vivas y los productos de la floricultura tienen una ventaja comparativa superior a la de otros capítulos arancelarios se realiza un análisis a través del tiempo para el periodo que comprende desde enero del 2005 hasta noviembre del 2019, a fin de proyectar las exportaciones por los siguientes 12 meses y brindar un panorama del desempeño de la partida arancelaria en los siguientes meses. La base de datos que se usó es la de las participaciones de exportaciones mensuales de plantas vivas y productos de la floricultura en las exportaciones mensuales totales de Colombia a Canadá, de manera que se obtuvo un total de 179 observaciones en unidades porcentuales.

Con el uso del software Eviews se define el modelo con polinomio de grado 5 y tres dummies: la primera correspondiente a febrero, la segunda al periodo de tiempo comprendido entre enero del 2013 hasta julio del mismo año, la tercera correspondiente al periodo de enero del 2016 hasta mayo del mismo año. Se ubican en estas fechas por la mayor participación de las exportaciones de la partida a causa de fechas célebres en Canadá, como, por ejemplo, San Valentín y el Día de la madre, entre otras. Con este modelo se comprueban los supuestos de normalidad, homocedasticidad, no autocorrelación y significancia de los coeficientes.

Luego de comprobar los supuestos en el software R Studio se importa la base de datos y se realiza el proceso de estimación y proyección de datos, usando, en primer lugar, la estimación y la proyección dentro de la muestra para obtener como resultado que el mejor pronóstico se realiza a partir del método suavizado Holt-Winters aditivo con un error MAE de 0,018 unidades de índice. 
En la tabla 11 se presenta la proyección para los 12 meses siguientes a noviembre de 2019.

Tabla 11. Proyección de exportaciones capítulo 06

\begin{tabular}{|c|c|c|}
\hline$\#$ & Fecha & Participación \\
\hline $\mathbf{1}$ & dic-19 & 0.05771215 \\
\hline $\mathbf{2}$ & ene-20 & 0.09159997 \\
\hline $\mathbf{3}$ & feb-20 & 0.11626830 \\
\hline $\mathbf{4}$ & mar-20 & 0.08102774 \\
\hline $\mathbf{5}$ & abr-20 & 0.07930508 \\
\hline $\mathbf{6}$ & may-20 & 0.10945336 \\
\hline $\mathbf{7}$ & jun-20 & 0.09094548 \\
\hline $\mathbf{8}$ & jul-20 & 0.09231232 \\
\hline $\mathbf{9}$ & ago-20 & 0.08376871 \\
\hline $\mathbf{1 0}$ & sep-20 & 0.07255259 \\
\hline $\mathbf{1 1}$ & oct-20 & 0.08179498 \\
\hline $\mathbf{1 2}$ & nov-20 & 0.07693307 \\
\hline
\end{tabular}

Fuente. Elaboración propia con resultados obtenidos de R Studio.

De acuerdo con estos resultados es posible decir que la participación mensual de las exportaciones del capítulo arancelario 06, correspondiente a plantas vivas y productos de la floricultura, es decir, el sector floricultora, continua con un comportamiento similar al de los años anteriores, lo que se traduce en una falta de aprovechamiento de las exportaciones de esta partida, pues la ventaja comparativa dada por el sector y la condición arancelaria de entrada de $0 \%$ podría ser aprovechada con un aumento significativo en las exportaciones a Canadá.

\section{Conclusiones}

A pesar de la existencia del TLC Colombia-Canadá que actualmente cuenta con aproximadamente el $98 \%$ del universo arancelario desgravado, es decir, $0 \%$ de arancel, Canadá no es un socio significativo para Colombia, pues tan solo representa el $2 \%$ del total de exportaciones colombianas. 
La entrada en vigencia del TLC Colombia-Canadá no aumenta las exportaciones de productos colombianos con valor agregado a este mercado, pues después de la entrada en vigencia del tratado bilateral en el 2011 los flujos de mercancía en cuanto a exportaciones a Canadá se han mantenido constantes: la participación de cada sector - con $0 \%$ de arancel-en los flujos totales hacia ese mercado no han aumentado significativamente. Por tanto, se puede decir que, en términos de exportaciones de Colombia a Canadá, no se ha aprovechado en gran proporción el acuerdo comercial vigente desde el 2011.

Colombia podría diversificar su oferta exportable con el fin de llegar a Canadá con más que materias primas. El país cuenta con una ventaja comparativa en el sector de plantas vivas y productos de la floricultura que no ha sido aprovechada, pues se ha mantenido constante la participación de este sector en el total de exportaciones mensuales de Colombia a Canadá. El sector de floricultura constituye entonces un potencial para aumentar la capacidad de exportación a Canadá.

\section{Referencias}

Banco Mundial. (2010). Indicadores comerciales. Recuperado de https://bit.ly/33Apewd

DANE. (2017). Glosario importaciones y exportaciones. Recuperado de https://bit.ly/3tzyDi3

Hernández-Sampieri, R. H. (2017). Metodología de la investigación (6ª ed.). México: Mcgraw-Hill.

Ministerio de Comercio. (2018). Acuerdos comerciales vigentes. Recuperado de https://bit.ly/3f9cS3t

Pole, K. (2009). Diseño de metodologías mixtas: una revisión de las estrategias para combinar metodologías cuantitativas y cualitativas. Renglones. Revista Arbitraba en Ciencias Sociales y Humanidades, (60), 37-42. Recuperado de https://bit.ly/3y2a4xF

Procolombia. (2016). Partidas arancelarias. Recuperado de https://bit.ly/3bjsHn1

TradeMap. (2018). Comercio bilateral Colombia-Canadá. Recuperado de https://bit.ly/2SxHDaA 